\title{
ePortfolio companion: getting junior doctors engaged with the ePortfolio
}

\author{
Authors: Aaron Koshy, ${ }^{\mathrm{A}}$ Neal Chauhan ${ }^{\mathrm{B}}$ and Twm Davies ${ }^{\mathrm{C}}$
}

\section{Aims}

To explore the issues surrounding engagement with the ePortfolio with an interest in developing a mobile app to increase the support available.

\section{Methods}

A cross-sectional survey in the form of an online questionnaire created on SurveyMonkey was sent to junior doctors working in two independent NHS trusts (Newcastle and Leicester) in 2015-16. Feedback led to the design, development and publication of a standalone iPhone mobile application made by junior doctors using computer programming skills.

\section{Results}

One-hundred and two complete responses were obtained from junior doctors at the two sites. The majority of respondents $(64 \%)$ reported a need for increased support on engaging with the ePortfolio system. A third (33\%) required months of exposure before developing familiarity with the platform and $86 \%$ required a process of trial and error. Almost all junior doctors (99\%) have a smartphone and rated features such as calendar integration and a notes page to store reflections as 4.1 and 3.8 out of 5, respectively, in terms of usefulness.

\section{Conclusion}

Completing the ePortfolio is a complex task for junior doctors that would benefit from additional support. It is clear with the availability of smartphones among peers that technology could be utilised to provide guidance. A tailor-made app with an inbuilt guide and series of video tutorials has been created for junior doctors to succeed in completing the NHS ePortfolio. Feedback from the questionnaire has led to the development of additional functionality such as a reminder system, logbook of teaching, storage for reflection and others. The app, ePortfolio Companion, was released for free with significant positive feedback and over

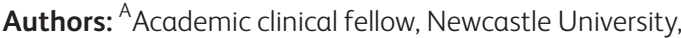
Newcastle, UK; ${ }^{B}$ Core medical trainee, Bristol Hospital, Bristol, UK; ${ }^{\mathrm{C}} \mathrm{GP}$ trainee, East of England, UK
300 downloads with its launch. It is known that educational needs are more effectively met through early engagement with the curriculum and annual review of competence progression. Further scope is available to improve quality and support in medical training through innovation and novel solutions. It is hoped in the near future to roll out the app across all existing ePortfolio systems with full integration so that junior doctors are able to seamlessly update and improve their ePortfolio efficiently in an engaging manner.

\section{Conflict of interest statement}

Authors have completed the foundation programme using the NES NHS ePortfolio systems.

\section{Disclosure of interest}

None declared. 